\title{
Vasa Previa
}

National Cancer Institute

\section{Source}

National Cancer Institute. Vasa Previa. NCI Thesaurus. Code C87127.

Umbilical blood vessels that have inserted into the amniotic membrane (membranous insertion) that in turn are above or adjacent to the cervical os and are at risk of rupture. 